\title{
PREPARATION AND EVALUATION OF SULFASALAZINE LOADED SODIUM ALGINATE MICROBEADS FOR SUSTAINED DELIVERY
}

\author{
JYOTHIKA MATTAM ${ }^{1}$, KRISHNA SAILAJA A ${ }^{2 *}$
}

${ }^{1}$ Department of Pharmaceutics, RBVRR Women's College of Pharmacy, Barkathpura, Hyderabad, Telangana, India. ${ }^{2}$ Department of Pharmaceutics, RBVRR Women's College of Pharmacy, Osmania University, Hyderabad, Telangana, India. Email: akskub@gmail.com

Received: 13 January 2016, Revised and Accepted: 20 January 2016

\section{ABSTRACT}

Objective: The main objective of the work was to prepare and evaluate sulfasalazine loaded sodium alginate microbeads for sustained delivery for the treatment of inflammatory bowel disease and rheumatoid arthritis. Sulfasalazine has crystalluria, thrombocytopenia, and megaloblastic anemia as side effects, so to reduce side effect microbeads were prepared.

Methods: The sulfasalazine microbeads were prepared by inotropic gelation method by optimizing process parameters such as concentration of calcium chloride, agitation speed, and time of agitation. The concentration of polymer sodium alginate was varied.

Result: Among the five formulations, the best formulation was considered by comparing process parameters such as the entrapment efficiency, drug content, in vitro drug release studies, scanning electron microscope analysis, and zeta potential.

Conclusion: On comparison, B3 formulation was considered as best formulation with a mean particle size ranging from 40.9 to $244 \mu \mathrm{m}$, drug content of $94.7 \%$, entrapment efficiency of $87.7 \%$, and the drug release was found to be $97.1 \%$ for 12 hrs and followed zero order kinetics and non-Fickian diffusional pathway, with a zeta potential value of $-56.8 \mathrm{mV}$ indicating higher stability.

Keywords: Inotropic gelation method, Sodium alginate, Microbeads, Rheumatoid arthritis, Side effects.

(C) 2016 The Authors. Published by Innovare Academic Sciences Pvt Ltd. This is an open access article under the CC BY license (http://creativecommons. org/licenses/by/4. 0/) DOI: http://dx.doi.org/10.22159/ajpcr.2016.v9s2.10701

\section{INTRODUCTION}

Controlled drug delivery systems are designed to deliver the drug at predetermined rate at a specific site thereby reducing the dose, dosing frequency, and side effects of the drug $[1,2]$. Controlled drug delivery system holds promising results in site-specific targeting. One of such approach is the formulation of microbeads as drug carriers. Microbeads are defined as the uniform polymeric particles, typically ranging in size from $0.5 \mu$ to $500 \mu \mathrm{m}$ in diameter [3]. Inotropic gelation method is based on the electrostatic interaction between amine groups of the polymer and negatively charged group of polyanion. Naturally occurring polysaccharides are used as biopolymers in novel drug delivery system, thus providing ecofriendly pharmaceutical process. Among the natural polymers, sodium alginate was considered to be the best polymer and forms a reticulated structure when cross-linked with polyvalent or divalent ions. Sulfasalazine was selected as a drug of choice for the preparation of sodium alginate microbeads. It belongs to a class of disease-modifying antirheumatic drugs used in the treatment of rheumatoid arthritis to slow down disease progression. Sulfasalazine is a sulfa drug, a derivative of mesalazine and prodrug of 5 aminosalicylic acid that is covalently linked to the antibiotic sulfapyridine by an azo bond. It has been used in the treatment of inflammatory bowel disease and rheumatoid arthritis because of its ability to induce T-lymphocyte apoptosis modulates inflammatory mediators. Sulfasalazine is available in the dosage form of salazopyrin $500 \mathrm{mg}$ tablets, salazopyrin enteric coated tablets. For treatment of rheumatoid arthritis, $500 \mathrm{mg}$ tablets are taken 2-3 times a day. As the oral route of solid dosage forms have many side effects and chances of missing the doses, sulfasalazine loaded sodium alginate microbeads were prepared, thereby to reduce the dose, dosing frequency, and side effects of the drug.

\section{MATERIALS AND METHODS}

Materials

Drug

Sulfasalazine (gift sample from posh chemicals).
Excipients

- Sodium alginate obtained from SD Fine-Chem Limited, Mumbai,

- Ethanol (SD Fine-Chem Limited, Mumbai),

- Calcium chloride (SD Fine-Chem Limited, Mumbai),

- Potassium dihydrogen phosphate (SD Fine-Chem Limited, Mumbai)

- $\quad$ Sodium hydroxide (SD Fine-Chem Limited, Mumbai).

\section{Methods}

\section{Preparation and evaluation of microbeads}

Sulfasalazine loaded microbeads were prepared by dissolving sodium alginate in distilled water at $800 \mathrm{rpm}$ for 30 minutes to get a bubble free clear solution of different concentrations (1\%,2\%,3\%, 2:1, and 3:1). Sulfasalazine was accurately weighed and added to the polymeric solution to form a clear solution. The counterion solution was prepared by dissolving $4 \%$ calcium chloride solution. The drug loaded polymeric solution was added dropwise using 20-gauge hypodermic needle fitted with a syringe into aqueous solution of polyvalent cations. The cations diffuse into the drug loaded polymeric drops, forming a three dimensional lattice of ionically cross-linked moiety. The formed microbeads were kept in calcium chloride solution for 30 minutes to undergo complete curing. The microbeads were filtered and air dried [4].

Five formulations of microspheres were prepared by varying the concentration of drug:polymer ratios (Table 1).

\section{Characterization of microbeads}

Compatibility studies Fourier transform infrared (FTIR) analysis The FTIR analysis of the sulfasalazine was carried out for qualitative compound identification. To check the compatibility of the drug with various polymers, IR spectra of drug, polymers, and combination of the drug and polymers were taken on an FTIR spectrophotometer in the wave number region of 4000-400/cm. The IR spectra of drug, polymers, and their combination are shown in spectra. 
Study of surface morphology by scanning electron microscope (SEM) The prepared formulations were dispersed in deionized water and sonicated for 30 minutes. A circular metal plate is taken onto which carbon double tape $(1 \mathrm{~mm} \times 1 \mathrm{~mm})$ is stickered; a drop of the resultant dispersion is placed onto the tape and allowed to dry for a while. Then, it is scanned under SEM for morphology.

\section{Zeta potential measurement}

The zeta potential (surface charge), which indicates the stability of the microbeads, can be defined as electrokinetic potential that is determined by electrophoretic mobility. The sample was prepared by diluting with doubled distilled water and corresponding zeta potential measured using Malvern Zetasizer.

Product yield

The yield of the prepared formulations was calculated as the percentage of the weight of the dried product at room temperature compared to the theoretical amount. Production yield is calculated using the following equation:

Product yield $=\frac{\text { Weight of the product }}{\text { Weight of raw materials }} \times 100$

Drug content

The various batches of the formulations were subjected for drug content analysis. Accurately weighed microsphere samples were mechanically powdered. The powdered microspheres were dissolved in adequate quantity of pH 7.4 phosphate buffer in two-necked round bottomed

Table 1: List of formulations of microbeads

\begin{tabular}{lll}
\hline Serial number & Formulations & Drug:Polymer ratio \\
\hline 1 & B1 & $1: 1$ \\
2 & B2 & $1: 2$ \\
3 & B3 & $1: 3$ \\
4 & B4 & $2: 1$ \\
5 & B5 & $3: 1$ \\
\hline
\end{tabular}

flask. With the help of mechanical stirrer, it was allowed to stir for $3 \mathrm{hrs}$ then filter. The ultraviolet (UV) absorbance of the filtrate was measured using a UV spectrometer at $359 \mathrm{~nm}$.

The drug content for the formulations was determined by calculating:

Drug content $=\frac{\text { Practical drug content }}{\text { Theoretical drug content }} \times 100$

Entrapment efficiency

The various batches of the formulations were subjected for entrapment efficiency. Accurately weighed microsphere samples were added in adequate quantity of $\mathrm{pH} 7.4$ phosphate buffer and were centrifuged in ultracentrifuge at $17,240 \mathrm{rpm}$ at $-4^{\circ} \mathrm{C}$ for 40 minutes. The free drug concentration was determined spectrophotometrically at $359 \mathrm{~nm}$.

The entrapment efficiency for all the formulations was calculated by:

Amount of microcapsules taken

Entrapment efficiency $=\frac{\text {-unentrapped drug }}{\text { Amount of microcapsules taken }} \times 100$

\section{In vitro drug release studies}

The in vitro drug release studies for sulfasalazine formulations were carried out for 12 hrs using USP Type II dissolution apparatus (paddle type). Dissolution medium used was phosphate buffer (pH 7.4), each $900 \mathrm{ml}$ and temperature was maintained at $37^{\circ} \mathrm{C} \pm 2{ }^{\circ} \mathrm{C}$ at $50 \mathrm{rpm}$. Sulfasalazine formulations equivalent to $50 \mathrm{mg}$ was used for each dissolution study. $5 \mathrm{ml}$ samples were collected periodically and replaced with a fresh $5 \mathrm{ml}$ of $\mathrm{pH} 7.4$ phosphate buffer. The concentration of sulfasalazine was determined spectrophotometrically at $359 \mathrm{~nm}$ by suitable dilutions.

\section{RESULTS AND DISCUSSION}

\section{FTIR spectrum}

The prepared five formulations were characterized for drug-polymer interactions using FTIR (Horiba Scientific, Ltd.).

The peaks obtained in the spectra correlated with the peaks of drug spectrum. $1676 / \mathrm{cm}$ indicating the presence of carboxyl group. 1200-1120/cm indicating the presence of sulfonyl group. There

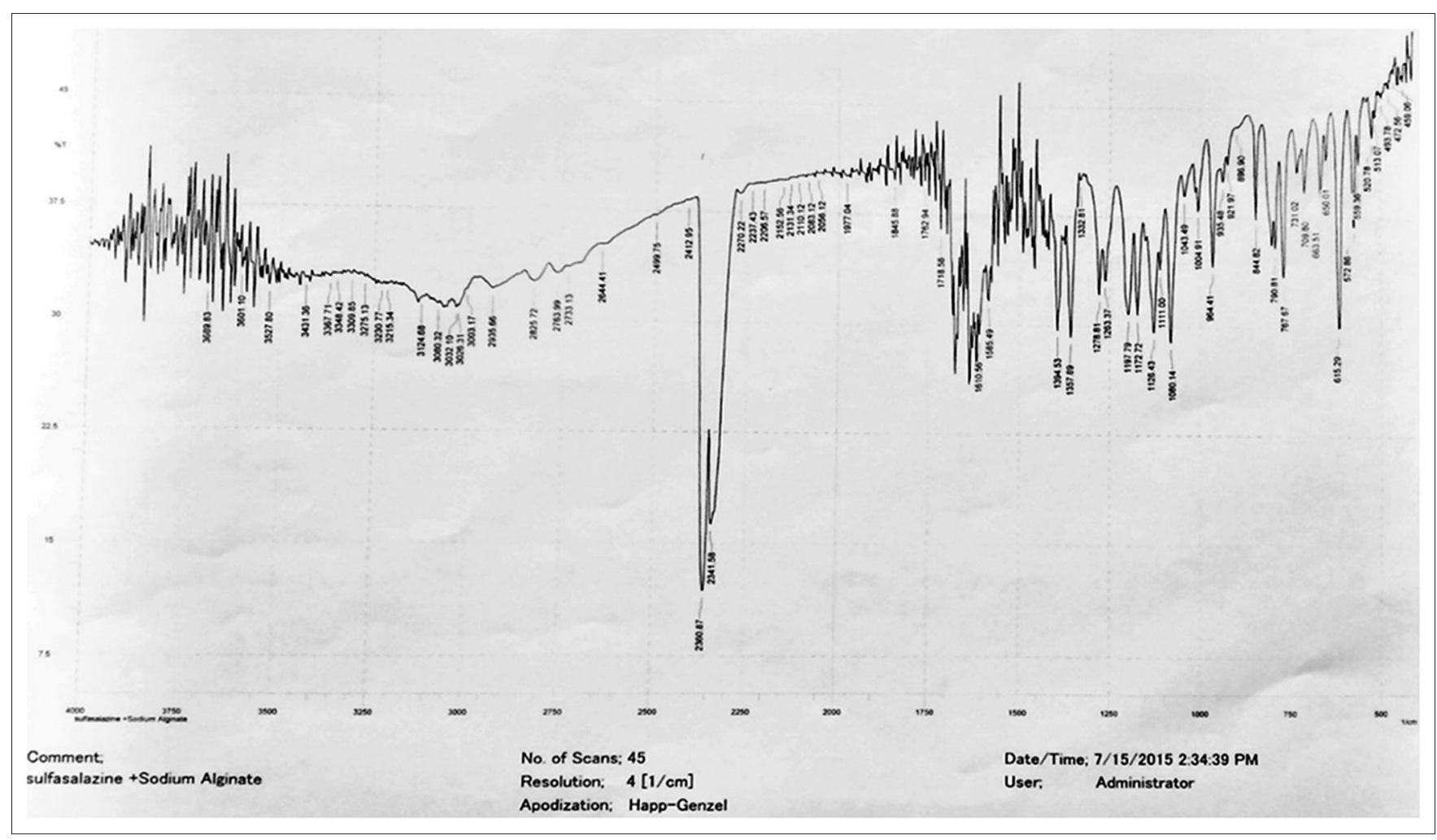

Fig. 1: Fourier transform infrared spectrum of best formulation of microbeads 


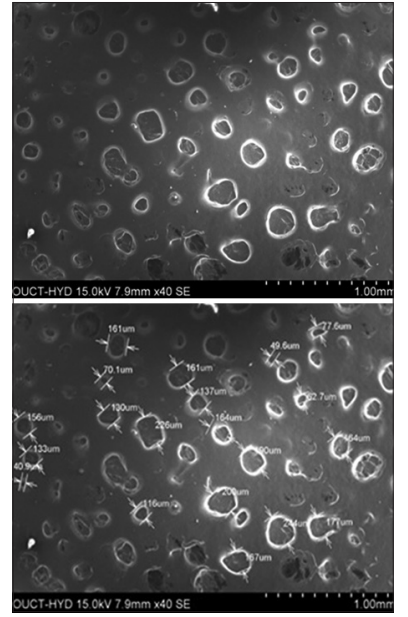

Fig. 2: Scanning electron microscope images of best formulation of microbeads were no physical or chemical interactions and the peaks obtained in the spectra's of each formulation correlated with the peaks of drug spectrum (Fig. 1).

SEM

Surface morphology was determined for all five formulations using SEM (S-3700N, Hitachi, Japan).

The SEM images were found to be spherical with size ranging from 40.9 to $244 \mu \mathrm{m}$ (Fig. 2).

\section{Zeta potential}

The best formulation of microbeads was characterized for zeta size to determine the stability of the formulation.

From Fig. 3, the zeta potential was found to be $-56.8 \mathrm{mV}$ indicating higher stability.

\section{Product yield}

Sulfasalazine microbeads were prepared by inotropic gelation method. After filtration, the obtained microbeads were evaluated for product yield.

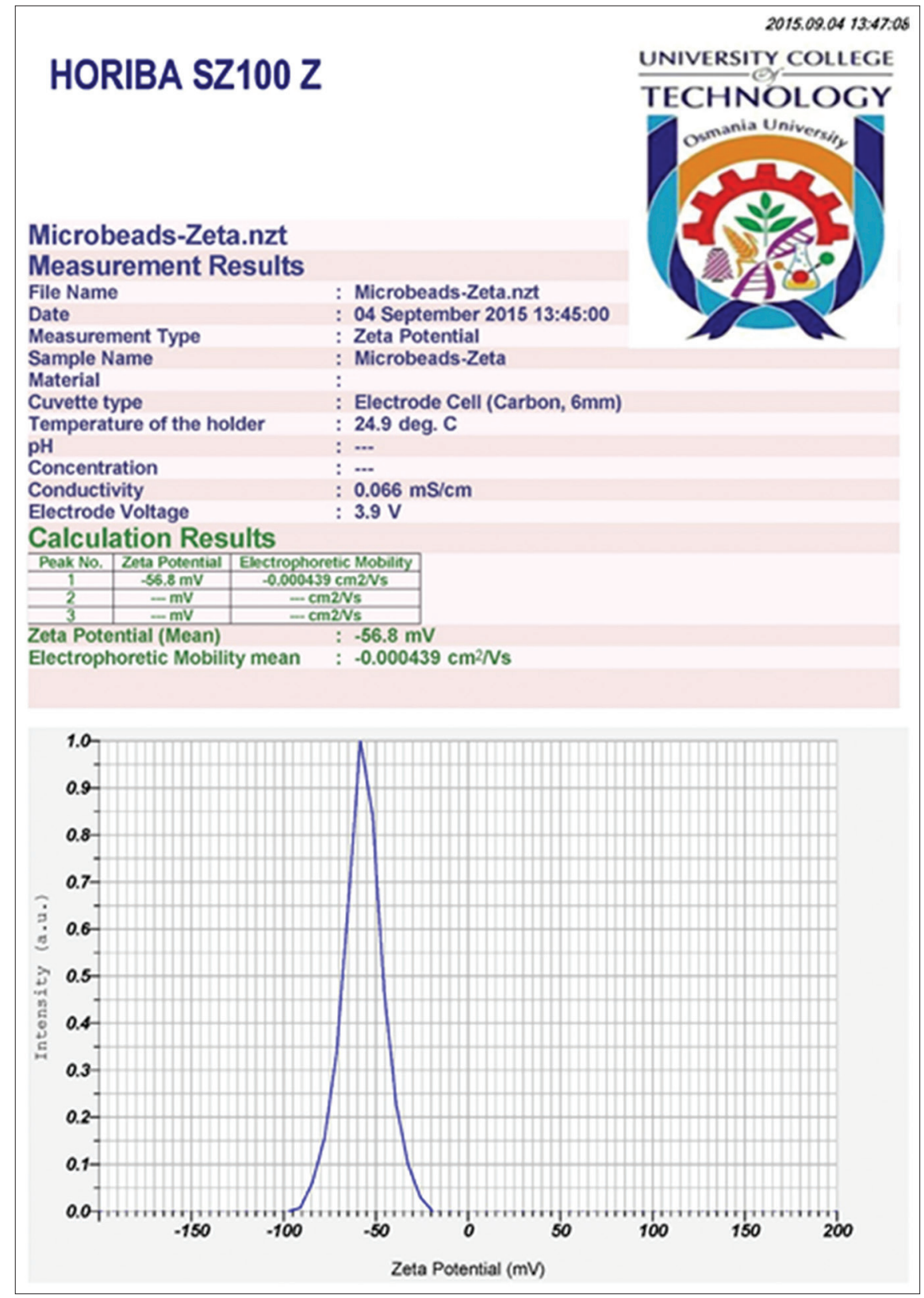

Fig. 3: Zeta potential report of best formulation B3 microbeads 
The product yield of prepared five formulations B1, B2, B3, B4, and B5 was found to be $90 \%, 91 \%, 97 \%, 86 \%$, and $84 \%$, respectively. Out of the five formulations, the highest product yield was observed for B3 formulation (Fig. 4).

\section{Drug content}

The prepared microbeads formulations were evaluated for drug content. Drug content of B1, B2, B3, B4, and B5 was found to be $82 \%, 90.8 \%$, $94.7 \%, 79.3 \%$, and $75 \%$, respectively. Out of the five formulations, the higher drug content was observed for B3 formulation (Fig. 5).

\section{Entrapment efficiency}

The entrapment efficiency of all the formulations of microbeads was calculated.

Entrapment efficiency of B1, B2, B3, B4, and B5 was found to be $77.6 \%$, $84.4 \%, 87.7 \%, 68 \%$, and $66.6 \%$, respectively. Out of the five formulations, the highest entrapment efficiency was observed for B3 formulation (Fig. 6).

\section{In vitro release studies}

In vitro drug release studies were performed using USP dissolution apparatus Type 2 paddle apparatus. The study was conducted for $12 \mathrm{hrs}$.

From the data, it was observed that B1 formulation showed $87.4 \%$ of drug release within 6 hrs and from B2 formulation $98.07 \%$ of drug release was observed within 8 hrs. From B3 formulation, 97.1\% of drug release was observed within a period of $12 \mathrm{hrs}$. B4 formulation showed $91.16 \%$ of drug release in 4 hrs and B5 showed 94.4\% in 4 hrs (Fig. 7).

With increase in polymer concentration, the sustain release profile of the formulation was found to be increased. The burst release was observed due to the drug present on the surrounding wall of the microbeads.

Comparison of best formulation with various kinetic models Several plots (zero order plot, first order plot, Higuchi plot, and Peppas plots) were drawn to know the release kinetics and drug release mechanism.

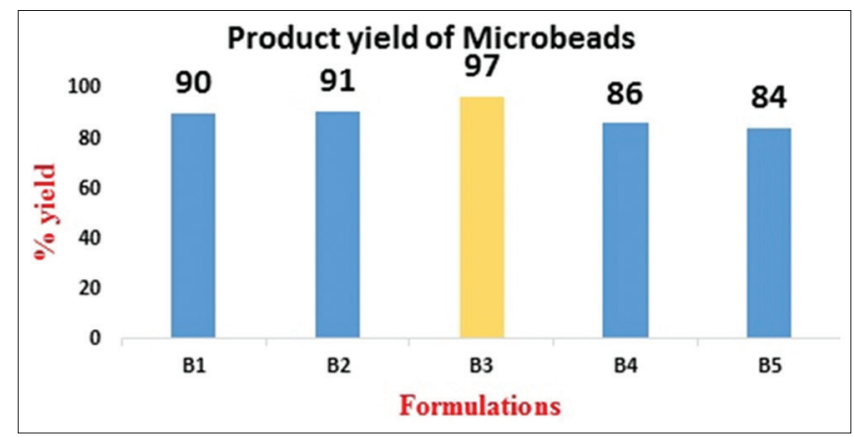

Fig. 4: Comparison of product yield among the five formulations of sulfasalazine microbeads

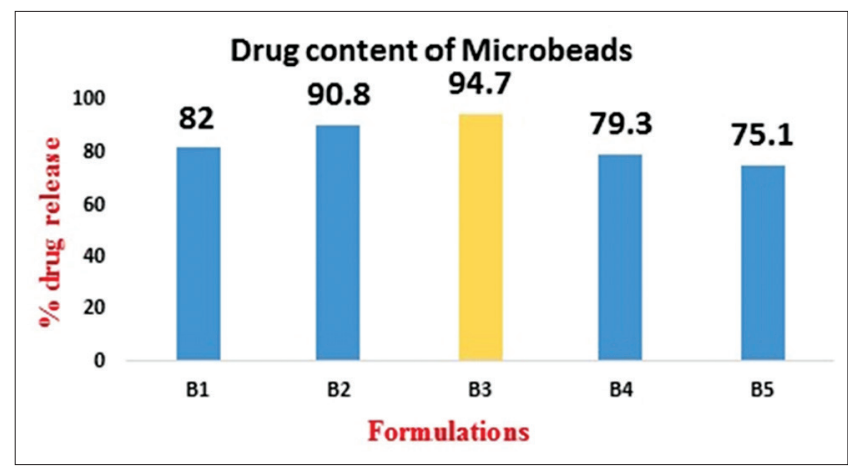

Fig. 5: Comparison of drug content among the five formulations of sulfasalazine microbeads
From the results, it was concluded that the drug release was following zero order kinetics with non-Fickian diffusional pathway (Fig. 8).

\section{DISCUSSION}

The formulation of sulfasalazine loaded microbeads for the treatment of rheumatoid arthritis was prepared using sodium alginate as the sustained release polymer and calcium chloride as a cross-linking agent [5]. The inotropic gelation technique was used to prepare microbeads, in which the gelation of anionic polysaccharide (sodium alginate) was achieved with oppositely charged counterion, i.e., calcium $\left(\mathrm{Ca}^{2+}\right)$ ions to form microbeads [6]. The gelation of alginate was caused by the formation of egg box junction to associate divalent metal ions of alginate polymer chain. The particle size of microbeads was found to be 40.9-244 $\mu \mathrm{m}$, respectively. It was observed that the particle size increases with increase in polymer sodium alginate concentration or by varying the exposure time to calcium chloride. A significant increase in the percentage entrapment efficiency was observed with increase in polymer concentration and calcium chloride concentration, but polymer concentration was more effective. In vitro dissolution studies were carried out by USP Type II apparatus in phosphate buffer of $\mathrm{pH} 7.4$ for $12 \mathrm{hrs}$. The sulfasalazine loaded sodium alginate microbeads demonstrated a drug release of $97.1 \%$ for $12 \mathrm{hrs}$ for B3 formulation. The release of sulfasalazine from microbeads reveals more sustained release nature with an increase in sodium alginate concentration. The results clearly indicate that the increase in apparent cross-linking density delays the alginate gel disintegration in phosphate buffer ( $\mathrm{pH}$ 7.4) due to retardation of calcium $\left(\mathrm{Ca}^{2+}\right)$ ions exchange with sodium $\left(\mathrm{Na}^{+}\right)$and eventually lead to increase in lag time. The in vitro release data of sulfasalazine was processed to understand the linear relationship which follows zero order of kinetics because it shows highest regression value of 0.9354 [7].

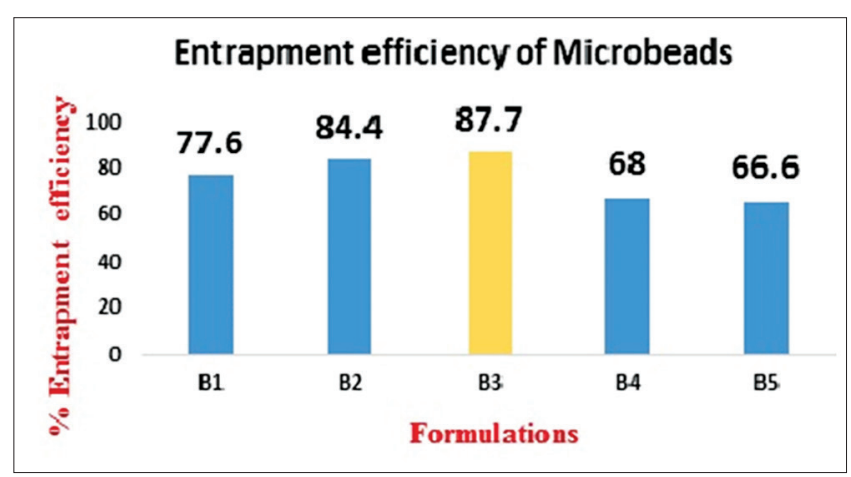

Fig. 6: Comparison of entrapment efficiency among the five formulations of sulfasalazine microbeads

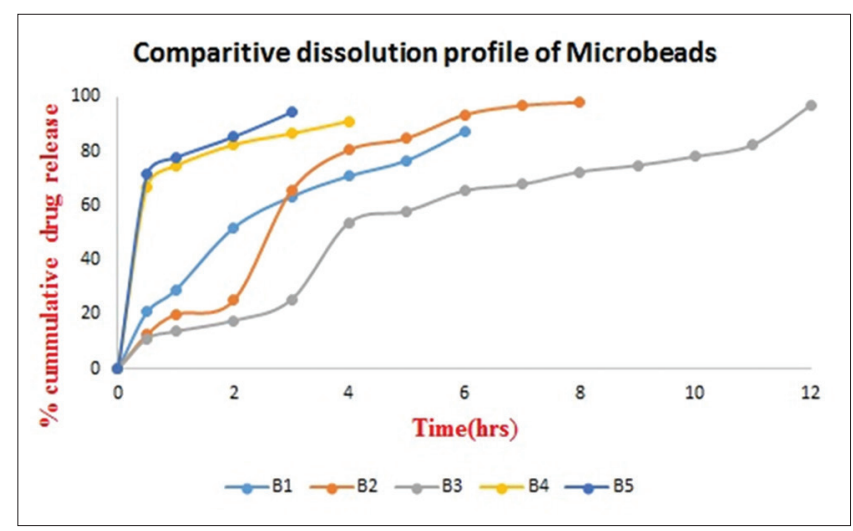

Fig. 7: Comparative in vitro drug release of sulfasalazine microcapsules 


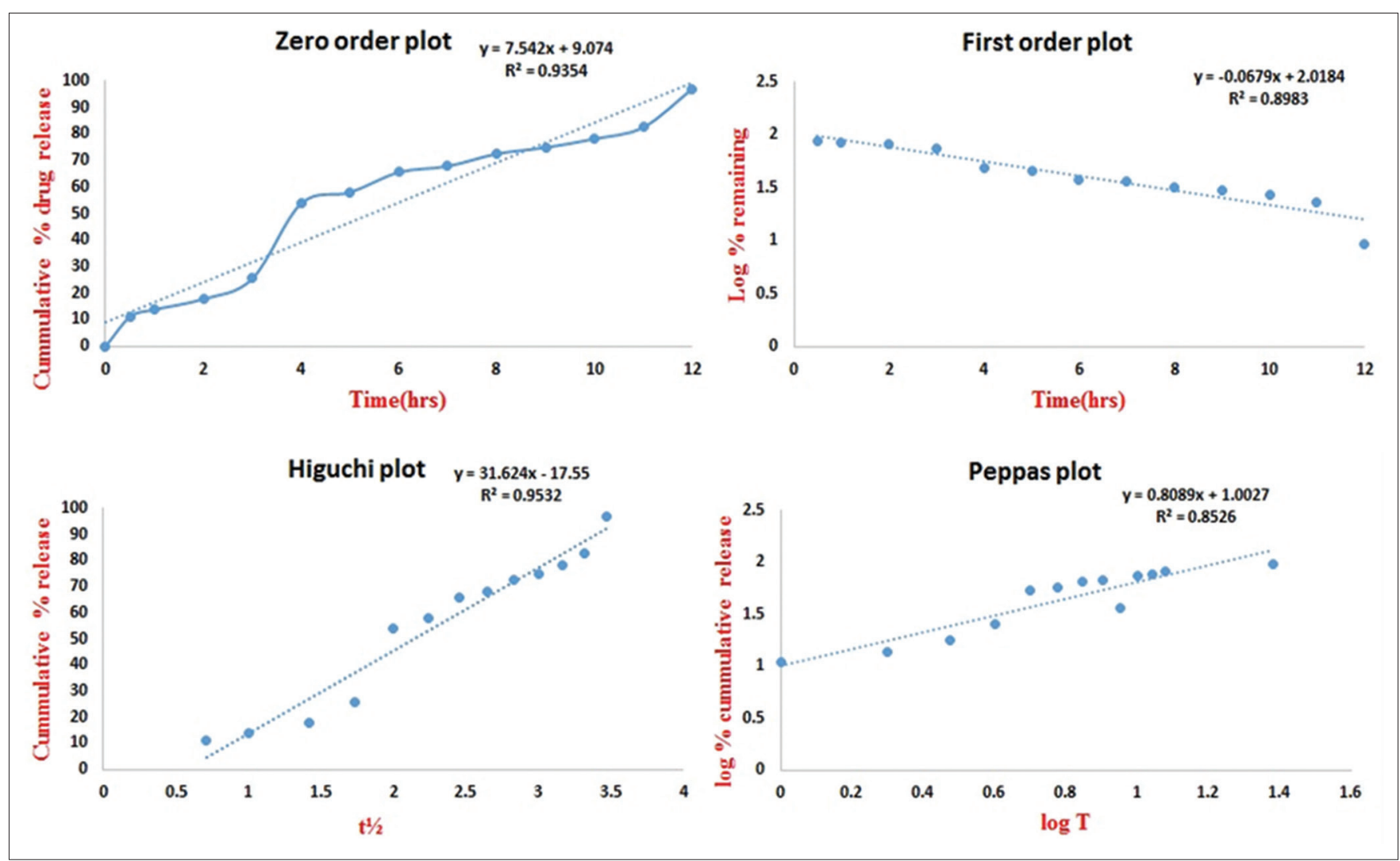

Fig. 8: In vitro drug release plots of best formulation of sulfasalazine microbeads

According to Korsmeyer-Peppas equation, the value of $n$ is 0.8089 which is more than 0.5 which indicates that it follows non-Fickian diffusion. Zeta potential value of $-56.8 \mathrm{mV}$ indicated high negative surface charge on microbeads which, in turn, indicates higher stability [8].

\section{CONCLUSION}

Inotropic gelation technique can be used for the preparation of sulfasalazine loaded sodium alginate microbeads using calcium chloride as cross-linking agent. The prepared microbeads showed higher drug entrapment and drug content. Sulfasalazine release from microbeads was influenced by sodium alginate (sustained release polymer).The microbeads were prepared without the use of organic solvents. Data for in vitro drug release indicated sustained release behavior. By fitting the data in various kinetic models, it was found that it exhibit first order kinetics followed by non-Fickian diffusion. Microbeads of sulfasalazine decrease the incidence of side effects and improve patient compliance thereby reducing the dose and dosing frequency.

\section{ACKNOWLEDGMENT}

The authors would like to thank RBVRR Women's College of Pharmacy, Principal Dr. M. Sumakanth, for providing funds for the work. The authors would like to acknowledge Mrs. K. Sumalatha and Mrs. D. Suvarna, for providing us technical assistance.

\section{REFERENCES}

1. Christina E. Preparation of microspheres of diclofenac sodium by inotropic gelation technique. Int J Pharm Pharm Sci 2013;5(1):228-1.

2. Deveswaran R, Manavalan R, Madhavan V, Bharath S. Formulation and evaluation of albumin microspheres containing aceclofenac. Int $\mathrm{J}$ Pharm Sci Rev Res 2010;4(1):112-7.

3. Available from: https://www.en.wikipedia.org/wiki/Microbeads (research)

4. Malik RK, Malik P, Gulati N, Nagaich U. Fabrication and in vitro evaluation of mucoadhesive ondansetron hydrochloride beads for the management of emesis in chemotherapy. Int $\mathrm{J}$ pharm Invest 2013;3(1):42-6

5. Ramana BV, Parameswari CS, Triveni C, Arundhati T, Reddy OV, Nagarajan G. Formulation and evaluation of sodium alginate microbeads of simvastatin. Int J Pharm Pharm Sci 2013;5(3):410-6.

6. Patil P, Chavanka D, Wagh M. A review on inotropic gelation method Novel approach for controlled gastroretentive gelispheres. Int J Pharm Pharm Sci 2012;4 Suppl 4:27-2.

7. Panda S, Pattnaik S, Maharana L, Botta GB, Mohapatra P. Formulation and evaluation of zidovudine loaded olibanum resin microcapsules: Exploring the use of natural resins as biodegradable polymeric materials for controlled release. Asian J Pharm Clin Res 2013;6(3):191-6.

8. Patel SR, Chand T, Maulik T. Formulation and evaluation of microspheres containing duloxetine hydrochloride. Int J Res Pharm Biomed Sci 2013;4(2):568-2. 